\title{
ENERGY EXPENDITURE AND INTENSITY OF INTERACTIVE VIDEO DAMCE GAMES ACCORDING TO HEALTH RECOMMENDATIONS
}

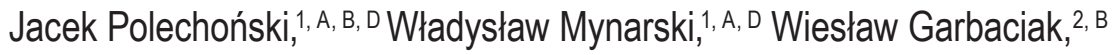 \\ Artur Fredyk, ${ }^{3, \text { B }}$ Michał Rozpara, ${ }^{1, C}$ Agnieszka Nawrocka ${ }^{1, A, D}$ \\ 1 Department of Tourism and Health-Related Physical Activity, The Jerzy Kukuczka Academy of Physical Education in Katowice, Poland \\ ${ }^{2}$ Department of Theory and Methodology of Physical Education, The Jerzy Kukuczka Academy of Physical Education in Katowice, Poland \\ ${ }^{3}$ Faculty of Physical Education, The Jerzy Kukuczka Academy of Physical Education in Katowice, Poland \\ A Study Design; ${ }^{B}$ Data Collection; ${ }^{\mathrm{C}}$ Statistical Analysis; ${ }^{\mathrm{D}}$ Manuscript Preparation; ${ }^{\mathrm{E}}$ Founds Collection
}

\author{
Address for correspondence: \\ Agnieszka Nawrocka \\ Department of Tourism and Health-Related Physical Activity, The Jerzy Kukuczka Academy of Physical Education in Katowice \\ Mikolowska 72a, 40-065 Katowice, Poland \\ E-mail: a.nawrocka@awf.katowice.pl
}

Ahstract Backround: The aim of this study was the assessment of energy expenditure (EE) and the intensity of physical activity (PA) of adult women during the interactive dance video game (IDVG) Dance Central on Xbox 360 Kinect in the context of health recommendations.

Methods: Twenty eight intentionally selected women (mean \pm SD age: $21.8 \pm 1.1$ years, body height: $167.3 \pm 6.1 \mathrm{~cm}$, body mass: $59.3 \pm 5.9 \mathrm{~kg}$, body mass index: $21.2 \pm 1.3$ ) met the inclusion criteria and took part in to the experiment. Heart rate monitor, accelerometer, and pedometer were used as measurement tools. Participants performed the same easy dance routine three times (for a total of $10 \mathrm{~min}$.). Trials consisted of imitating the motions presented by the virtual dancer.

Results: The data from accelerometer and heart rate monitor were similar, and indicated the moderate intensity of interactive dance video game (IDVG) Dance Central. However, energy expenditure assessed by pedometer was significantly lower.

Conclusion: IDVG Dance Central may be useful in increasing the daily dose of physical activity of adult women and meet the health-related recommendations provided its regular practice.

Key Worlls interactive dance video games, active video games, energy expenditure

\section{Introduction}

In the past few years, in the video game market, more and more so-called active video games (AVGs) appear, in which the user controls the game by the movements of the whole body. This leads to a much larger motor activation of the players than during the typical video games controlled by typical pointing devices. Player movements are similar to the real movements, and the participants' involvement is increased by the forms of competition used in games. 
Many authors see potential health and social benefits of participation in AVGs. It is confirmed by, among others, increasing number of research on effects of using this form of physical effort in physical education (Hayes, Silberman, 2007; Levac, Miller, 2013; Trout, Christie, 2007) treatment and physiotherapy (AlSaif, Alsenany, 2015; Howcroft et al., 2012; Kimhy et al., 2016; Miller, Hayes, Dye, Johnson, Meyers, 2012; Salonini et al., 2015) and promotion of health and physical activity (Biddiss, Irwin, 2010; Smallwood, Morris, Fallows, Buckley, 2012; Studenski et al., 2010; Taylor, Kerse, Frakking, Maddison, 2016).

In previous studies only one physical activity (PA) measuring tool was used. Meanwhile, the review indicates the legitimacy of the usage in the diagnosis of PA parameters several devices at the same time, which are recording both the physiological reactions of the organism to the physical effort (e.g. exercise heart rate, energy expenditure) and kinematic parameters of movements (e.g. the movement acceleration in the body axis, the number of steps) (Hills, Mokhtar, Byrne, 2014; Mynarski, Nawrocka, Rozpara, Cholewa, Tomik, 2013; Schutz, Weinsier, Hunter, 2001; Strath et al., 2013). Such a multifaceted PA diagnosis allows assessing the potential health benefits in reference to the different criteria of health-enhancing recommendations. Among these criteria, important are energy expenditure $(\mathrm{EE})$, exercise heart rate (HR), number of steps (NS) and intensity effort (IE).

The cognitive aim of the study was evaluation (diagnosis and assessment) of EE, HR, NS and IE during the interactive dance video game (IDVG) "Dance Central" among 21-year-old women, who were measured by different measuring devices (heart rate monitor, accelerometer, pedometer) and the confrontation of the results with the criteria of health-enhancing PA. We assume that IDVGs can be useful in increasing daily dose of physical activity if practiced regularly.

The usage of three measurement tools during the research was supposed to allow an initial estimation of diagnostic accuracy in the evaluation of the above-mentioned PA parameters while practicing IDVG. Presumably, data obtained with HR monitor and accelerometer during IDVG will be similar and will differ from data obtained using pedometer, because during IDVG there are few locomotive movements.

\section{Material and Methods}

\section{Participants}

The study involved 28 intentionally selected female students of the Academy of Physical Education in Katowice (see Table 1 for subject characteristics). The participants had no history of seizures or epilepsy, and they were informed about the product safety information. Participants were familiarized with the aim of monitoring of physical activity, the process of measurement and forms of the usage of its results. They did not have any previous experience with the IDVG used in this study. All participants were instructed on use of the Xbox's Kinect system and the Kinect dance game. Procedures were approved by the Research Ethics committee of the Jerzy Kukuczka Academy of Physical Education in Katowice.

Table 1. Participants characteristic

\begin{tabular}{lcl}
\hline \multicolumn{1}{c}{ Variables } & Mean & SD \\
\hline Age (years) & 21.8 & 1.1 \\
Body height $(\mathrm{cm})$ & 167.3 & 6.1 \\
Body mass $(\mathrm{kg})$ & 59.3 & 5.9 \\
BMl $\left(\mathrm{kg} / \mathrm{m}^{2}\right)$ & 21.2 & 1.3 \\
\hline
\end{tabular}




\section{Procedures}

To increase the reliability of the assessment of the results of physical activity monitoring during IDVG "Dance Central", measuring devices were used to record kinematic parameters of the movements (an accelerometer (Caltrac Monitor) and pedometer (Yamax SW-800)) and physiological parameters of the effort - pulsometer (Polar FT4). The first two devices were placed in a special belt worn on the hip of the participant. The receiver of the pulsometer was in the lower part of the forearm, above the wrist, and HR sensor was on the chest.

Before the research, participants' data were input into the memory of measurement tools; in the accelerometer: body weight and height, age, and gender; in pedometer: body weight and adopted by the manufacturer standard step length - $70 \mathrm{~cm}$; The pattern was taken from other authors (Groffik, Frömel, Pelclová, 2008). Birth date, gender, weight and height parameters were insert into HR monitor.

Three measurement tools obtained information about:

- absolute EE of undertaken physical exercise in kilocalories (kcal), estimated in the tool's memory on the basis of the acceleration of movements (accelerometer), recorded steps (pedometer) and exercise HR (Heart Rate Monitor),

- relative EE designated by recalculating the absolute EE per kilogram of participants' body weight; this indicator was treated as a dimension of the intensity of undertaken physical effort in metabolic equivalent units (MET) (Ainsworth et al., 2011).

The study was conducted before noon, in the laboratory room, on a specially prepared stand equipped with a display of 32 inches diagonal set at the eye level of players, and Microsoft Xbox 360 Kinect gaming console. Participants, while playing, were moving at a distance of about 2-3 $\mathrm{m}$ away from the monitor calibrated by the Kinect sensor cooperating with the console. They had space that allowed them to move freely their body and limbs.

Before the game, the one-minute course of IDVG Dance Central (DC) was demonstrated to each participant, and they were allowed to perform the 30-second try (part of the game). During the measurement, participants performed three times in one day the same dance routine to the song by Lady Gaga "Just Dance" in the "Perform It!" mode, on the "Easy" level of difficulty, excluding periods of inactivity between game loading time. The easiest difficulty level was chosen because students did not have previous experience with IDVG DC. Described research procedures gave a total of ten-minute continuous physical effort. The essence of $D C$ is the imitation of dance moves accurately and with the full amplitude, which are performed by a virtual dancer. The final result expressed in points depends on precise imitation of dance moves (similarity between player's moves and virtual pattern's moves).

\section{Statistical analysis}

For the statistical analysis, the Statistica v. 10 software (StatSoft Inc., USA) was used. Arithmetic Means (x), standard deviations (SD), and the differences between the mean values (d) were calculated. Oneway ANOVA with post-hoc t-tests was used to assess the statistical significance of differences between the results of participants' EE obtained with various measurement tools. The normality of data distribution was assessed with the Shapiro-Wilk test.

To compare EE during an interactive dance game to the selected criteria of health-enhancing PA according to the recommendations the World Health Organization (WHO) the following calculations were done:

- estimated for the measurement tools EE of 10-minute IDVG was converted into a one-hour effort in kcal, 
- EE in kcal was converted into kilogram per body weight, obtaining information about the individual intensity of undertaken physical effort in metabolic equivalent units (METs).

As health-enhancing was the PA of at least moderate intensity, assessed according the following criteria:

- physical effort within the intensity of 3-6 METs (kcal / kg / h) (WHO 2010),

- physical effort in the range of 50-69\% HR max (Strath et al. 2013),

- physical effort leading to do at least six thousand steps within one hour (steps/h) (Tudor- Locke et al., 2011).

\section{Results}

Studies indicated that the particular measurement tools identified the different value of absolute EE during the 10-minute DC game. It was demonstrated especially after the conversion of the results on the hour effort (Table 2). Similar results (within 90\%) were obtained by heart rate monitor $(249.9 \mathrm{kcal} / \mathrm{h})$ and accelerometer $(227.6 \mathrm{kcal} / \mathrm{h})$. Energy expenditure, estimated from the number of steps, was significantly lower $-166.4 \mathrm{kcal} / \mathrm{h}$. It was confirmed by the analysis of variance indicating the significant effect of the type of the measurement tool on the registered EE during IDVG $(p<0.01)$. Post-hoc tests indicate that EE estimated from the pedometer and heart rate monitor $(p<0.01)$ and the pedometer and accelerometer $(p<0.01)$ is significantly different (Table 2). Such difference does not occur between the results obtained by accelerometer and heart rate monitor.

Tahle 2. Absolute (kcal/h) and relative (METs) energy expenditure during interactive dance video game Dance Central on Xbox 360 Kinect

\begin{tabular}{lcccrrr} 
& Heart rate monitor & Accelerometer & Pedometer & $\mathrm{d}_{1}$ & $\mathrm{~d}_{2}$ & $\mathrm{~d}_{3}$ \\
\hline EE (kcal/h) & $249.9 \pm 115.0$ & $227.6 \pm 70.0$ & $166.4 \pm 54.2$ & 22.3 & $83.5^{*}$ & $61.2^{*}$ \\
EE (METs) & $4.2 \pm 1.9$ & $3.9 \pm 1.3$ & $2.8 \pm 0.9$ & 0.3 & $1.4^{*}$ & $1.1^{*}$ \\
\hline
\end{tabular}

EE - energy expenditure, MET - metabolic equivalents, d1 - the difference between the results obtained by accelerometer and heart rate monitor, d2 - the difference between the results obtained by heart rate monitor and pedometer, $\mathrm{d} 3$ - the difference between the results obtained by accelerometer and pedometer * - the difference statistically significant at level $p<0.01$.

The average intensity of physical effort during the DC game, estimated from the heart rate monitor (4.2 METs) and accelerometer (3.9 METs) do not differ significantly. Significantly lower intensity of physical effort (2.8 METs) was estimated on the basis of EE registered by the pedometer (Table 2).

The average intensity of physical effort estimated from the data obtained from the accelerometer and heart rate monitor was in the preferred (recommended) for health section of 3-6 METs, typical for the efforts of moderate intensity (WHO, 2010). Relative EE, estimated from the pedometer, identifies the intensity of IDVG physical effort on the low level ( $<3 \mathrm{METs}$ ), below the health benefits (Figure 1).

In subsequent analyses, results of physical effort heart rate $\left(\% H R_{\max }\right)$, used calories $(\mathrm{kcal} / \mathrm{kg} / \mathrm{h})$ and the number of steps (steps/h), obtained from the heart rate monitor, accelerometer and pedometer, during IDVG Dance Central on Xbox 360 Kinect, were referred to health standards recommended by other authors (Strath et al., 2013; Tudor-Locke et al., 2011). This approach to results shows that the accelerometer and heart rate monitor identify physical activity accompanying practicing IDVG DC as moderately intense, (Strath et al., 2013) and the pedometer - as low intense (Tudor-Locke et al., 2011) (Figure 2). 


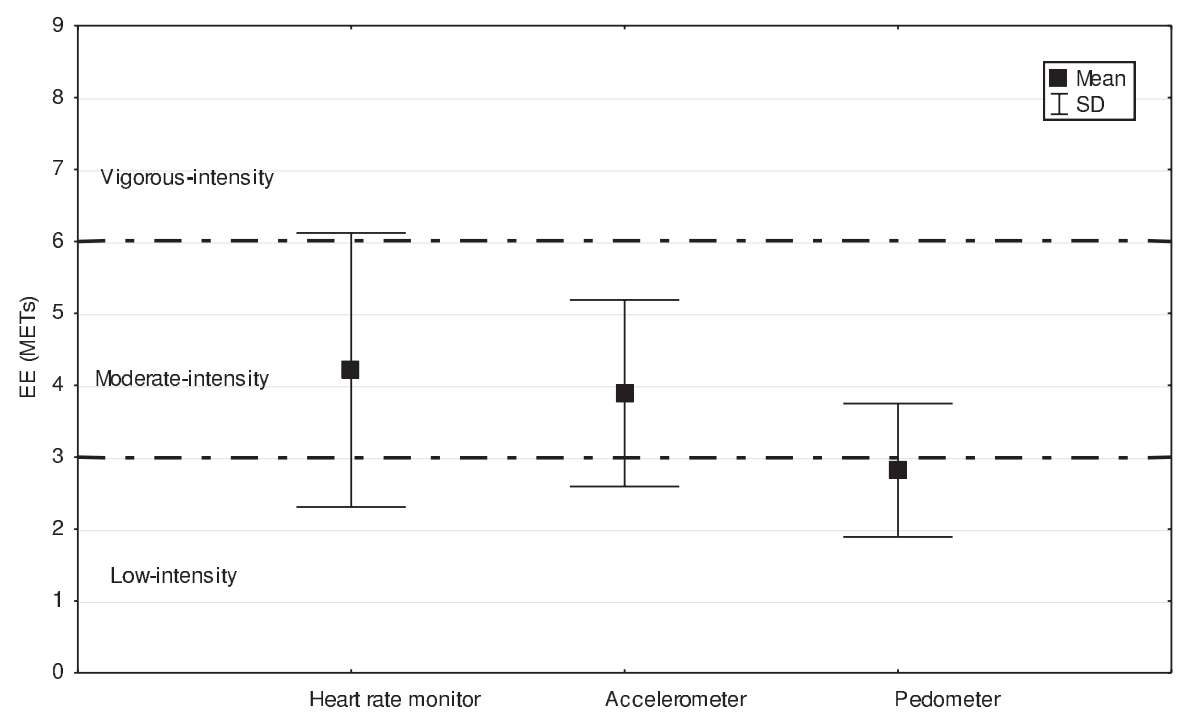

Figure 1. Relative (METs) energy expenditure of students during the interactive dance video game (IDVG) Dance Central (DC) on Xbox 360 Kinect, estimated with a heart rate monitor, accelerometer, and pedometer in the context to health recommendations

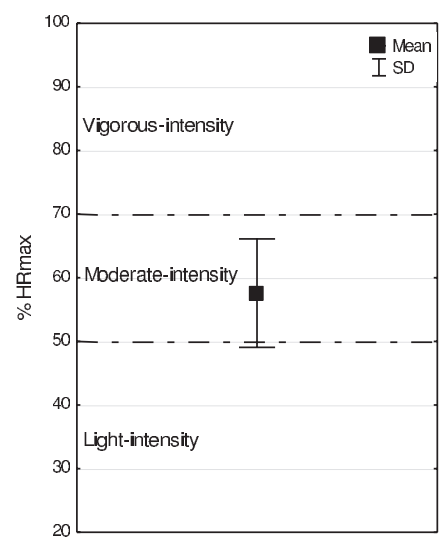

a)

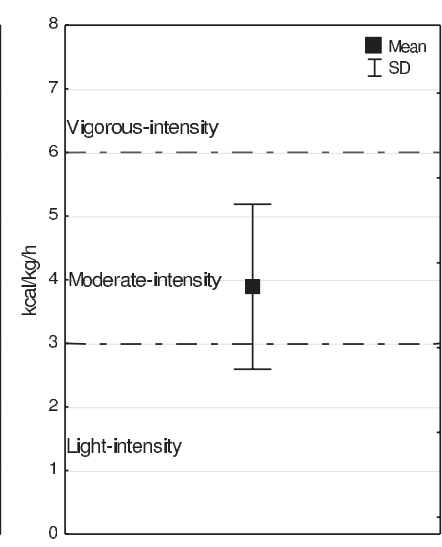

b)

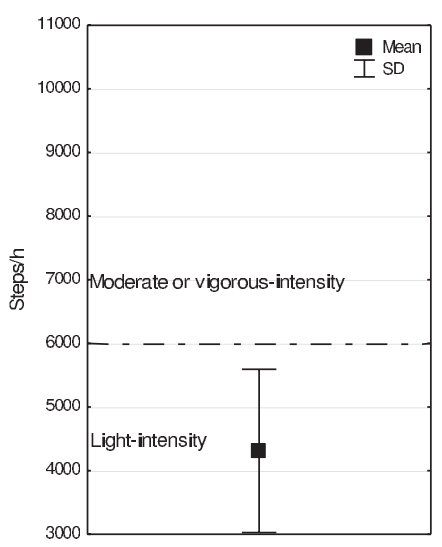

c)

Figure 2. Kinematic and physiological parameters characterizing the physical activity during the interactive dance video game (IDVG) Dance Central on Xbox 360 Kinect, estimated with a heart rate monitor (a), accelerometer (b) and pedometer (c) in the context to health recommendations 


\section{Discussion}

The aim of the research was to measure energy expenditure and intensity of physical effort during IDVGs DC practice by the students and preliminary assessment of the usefulness of measurement tools (accelerometer, strain gauge, and a pedometer) selected for this purpose.

The study showed the significant similarity between the results of the estimated EE of an hour practicing of IDVGs gained from the accelerometer $(249 \mathrm{kcal} / \mathrm{h})$ and heart rate monitor $(227 \mathrm{kcal} / \mathrm{h})$ and significantly lower from the pedometer $(166 \mathrm{kcal} / \mathrm{h})$.

The results recorded by the pedometer allow to accept the intensity of physical effort of IDVG players as low. The number of steps converted into an hour of the effort is clearly below the limit of moderate intensity, which is set on the level of 6,000 steps/h (Tudor-Locke et al., 2011). It allows to assume that the pedometer lowers energy expenditure during IDVG DC. It does not estimate EE because it records only the shocks caused by the steps, whereas while dancing there are movements around the torso and shoulder girdle with a particular EE. The research performed by Polechoński et al. (Polechoński, Mynarski, Nawrocka, 2015) suggests that a similar situation occurred in the case of Nordic Walking (NW). In some cases, the use of the pedometer to evaluate EE during the IDVGs may, however, be justified (Maloney, Threlkeld, Cook, 2012). It applies, for example, to an earlier version of IDVGs, such as Dance Dance Revolution (DDR), which involves putting feet (trampling) on special mats or platforms to the music and in accordance with the moving symbols (arrows) on the screen. In this case, the movement of the arms and torso does not matter. What counts are primarily movements of the lower limbs. Thus, according to Lin (2015) traditional dance games involve performing dance steps on the mat, modern IDVGs highlight the specific character of the dance moves.

The average intensity of physical effort during IDVG DC, assessed with the accelerometer and a heart rate monitor, ranged from 3 to 6 METs, typical for moderate intensive efforts (WHO, 2010). Similarly was in the case of the assessment of physical effort intensity on the basis of the \% Hrmax (Strath et al., 2013). Furthermore, EE and the physical effort intensity estimated with a heart rate monitor and accelerometer did not differ statistically significantly. It can be assumed that both measurement tools have similar diagnostic value in the assessment of $E E$ accompanying computer dance game practicing. This conclusion is eligible because the similarity between $\mathrm{EE}$ and physical effort intensity was presented, which were estimated on the basis of both physiological parameters of effort (heart rate monitor) and biomechanical characteristics (accelerometer). However, in study performed by Tripette et al. (2014) AVG intensities have been slightly but significantly underestimated by the accelerometer-based monitor compared to the indirect calorimetry.

Practicing IDVG DC is related to physical efforts. However, it should be remembered that such an effort will be beneficial for the health of people who practice it when it is properly distributed in time. According to WHO experts, adults (18-65 years) should, among others, perform physical exercises or other physical efforts of moderate intensity (3-6 METs) for a minimum of 150 minutes per week. Under this assumption, the efforts of the abovementioned intensity should last at least 30 minutes (WHO, 2010). Therefore, the DC IDVG could be useful in increasing the daily dose of physical effort and have health benefits when it is undertaken several times a week for several dozens of minutes (e.g., $5 \times 30$ min.).

Although the physical activity during IDVG DC fulfills the health-enhancing recommendations regarding physical effort intensity, in comparison with classical forms of dance and movement ranks on a low level (Ainsworth et al., 2011). Regarding the intensity, it a heads only some of the Caribbean dances and slow ballroom dances such 
as the waltz, foxtrot, tango. It should be emphasized, however, that the intensity of physical effort during IDVG DC would be higher when playing at a higher level than „Easy”. It is pointed in studies conducted by Noah et al. (Noah, Spierer, Tachibana, Bronner, 2011) during IDVG DDR in a group of adults (from 18 to 53 years), which show that during the game at the "Heavy" level, the average intensity of physical effort was 8 METs, and energy expenditure was $9 \mathrm{kcal} \cdot \mathrm{min}^{-1}$. Other studies show that higher motivation and physical effort can also be obtained by introducing the element of competition in AVGs in multiplayer mode (McGuire, Willems, 2015). Used in the present study, IDVG DC enables, among others, the usage of this mode.

According to Epstein et al. (Epstein, Beecher, Graf, Roemmich, 2007), IDVGs motivate children to physical effort, which seems to be the consequence of a natural fascination of the young generation with the "virtual world". Surprising is the fact that this also applies to adults. The study of 40 postmenopausal women (57 \pm 5 years) playing IDVGs shows that among the benefits of recreational practicing of such physical activity they see mostly entertainment values and the improvement of coordination. The participants also pointed to the body weight reduction, and to the fact that game is a challenge that encourages progress) (Inzitari, Greenlee, Hess, Perera, Studenski, 2009).

The positive influence of IDVGs on functional efficiency of middle-aged and older people speaks for the use of IDVGs as a beneficial form of physical activity. Mejia-Downs et al. (2011) during the six-week study with the usage of IDVGs, noticed the improvement of cardio-respiratory efficiency (cardiorespiratory status) measured with the ability to absorb oxygen and to reduce the BMI of adults. The participants stated that the IVDGs program was a good workout and motivated them to begin and continue the physical effort. As many as $40 \%$ of participants noticed better sleep and nearly half of the participants was considering the purchase of IVDGs. Pichierri, Murer, de Bruin (2012) observed the improvement of walk parameters among elderly people after a 12-week training program supplemented with IVDGs in comparison to a typical training program without such games. Chuang et al. (2015) argue that IDVGs DDR may improve cognitive functions in older women. Smith et al. (Smith, Sherrington, Studenski, Schoene, Lord, 2011) express the belief that IDVGs DDR can be a low budget home training method of older people and increase their involvement in the physical effort.

Our results and conducted discussion point the need of further, in-depth research on the caloric cost and intensity of physical activity associated with IDVGs practicing. In comparison to pedometers, more useful for this purpose are techniques of accelerometers and pulsometers. The value of these techniques in the assessment of varying IDVGs parameters may be clearly assessed only when confronted with the results of oxygen exchange during physical effort. The results of such research may also be useful for potential buyers of IDVG game console and enable them to choose the right interactive software for planning and undertaking the physical activity of a health-enhancing workout.

\section{Conclusions}

1. Playing IDVG Dance Central on the difficulty of "Easy” by women without experience in this area is characterized by a moderate intensity of physical effort.

2. IDVG Dance Central is a specific form of dance-movement and can be useful in increasing daily dose of physical activity and comply with the health recommendations if practiced regularly.

3. Data obtained with HR monitor and accelerometer during Dance Central IDVG are similar. 


\section{References}

Ainsworth, B.E., Haskell, W.L., Herrmann, S.D., Meckes, N., Bassett, D.R., Jr., Tudor-Locke, C., Greer, J.L., Vezina, J., Whitt-Glover, M.C., Leon, A.S. (2011). 2011 Compendium of Physical Activities: a second update of codes and MET values. Medicine and Science in Sports and Exercise, 43 (8), 1575-1581. DOI: 10.1249/MSS.0b013e31821ece12.

AlSaif, A.A., Alsenany, S. (2015). Effects of interactive games on motor performance in children with spastic cerebral palsy. J Phys Ther Sci, 27 (6), 2001-2003. DOI: 10.1589/jpts.27.2001.

Biddiss, E., Irwin, J. (2010). Active video games to promote physical activity in children and youth: a systematic review. Archives of Pediatrics and Adolescent Medicine, 164 (7), 664-672. DOI: 10.1001/archpediatrics.2010.104.

Chuang, L.Y., Hung, H.Y., Huang, C.J., Chang, Y.K., Hung, T.M. (2015). A 3-month intervention of Dance Dance Revolution improves interference control in elderly females: a preliminary investigation. Exp Brain Res, 233 (4), 1181-1188. DOI: 10.1007/ s00221-015-4196-x.

Epstein, L.H., Beecher, M.D., Graf, J.L., Roemmich, J.N. (2007). Choice of interactive dance and bicycle games in overweight and nonoverweight youth. Annals of Behavioral Medicine, 33 (2), 124-131. DOI: 10.1080/08836610701307835.

Groffik, D., Frömel, K., Pelclová, J. (2008). Pedometers as a method for modification of physical activity in students. J Hum Kinet, 20 , 131-138.

Hayes, E., Silberman, L. (2007). Incorporating video games into physical education. Journal of Physical Education, Recreation \& Dance, 78 (3), 18-24.

Hills, A.P., Mokhtar, N., Byrne, N.M. (2014). Assessment of physical activity and energy expenditure: an overview of objective measures. Frontiers in nutrition, 1,5 .

Howcroft, J., Klejman, S., Fehlings, D., Wright, V., Zabjek, K., Andrysek, J., Biddiss, E. (2012). Active video game play in children with cerebral palsy: potential for physical activity promotion and rehabilitation therapies. Archives of Physical Medicine and Rehabilitation, 93 (8), 1448-1456.

Inzitari, M., Greenlee, A., Hess, R., Perera, S., Studenski, S.A. (2009). Attitudes of postmenopausal women toward interactive video dance for exercise. J Womens Health (Larchmt), 18 (8), 1239-1243. DOI: 10.1089/jwh.2008.1176.

Kimhy, D., Khan, S., Ayanrouh, L., Chang, R.W., Hansen, M.C., Lister, A., Ballon, J.S., Vakhrusheva, J., Armstrong, H.F., Bartels, M.N., Sloan, R.P. (2016). Use of Active-Play Video Games to Enhance Aerobic Fitness in Schizophrenia: Feasibility, Safety, and Adherence. Psychiatric Services, 67 (2), 240-243. DOI: 10.1176/appi.ps.201400523.

Levac, D.E., Miller, P.A. (2013). Integrating virtual reality video games into practice: clinicians' experiences. Physiotherapy Theory and Practice, 29 (7), 504-512. DOI: 10.3109/09593985.2012.762078.

Lin, J.H. (2015). "Just Dance": The effects of exergame feedback and controller use on physical activity and psychological outcomes. Games Health J, 4 (3), 183-189.

Maloney, A.E., Threlkeld, K.A., Cook, W.L. (2012). Comparative effectiveness of a 12-week physical activity intervention for overweight and obese youth: Exergaming with "dance dance revolution". GAMES FOR HEALTH: Research, Development, and Clinical Applications, 1 (2), 96-103.

McGuire, S., Willems, M.E. (2015). Physiological Responses During Multiplay Exergaming in Young Adult Males are Game-Dependent. J Hum Kinet, 46, 263-271. DOI: 10.1515/hukin-2015-0054.

Mejia-Downs, A., Fruth, S.J., Clifford, A., Hine, S., Huckstep, J., Merkel, H., Wilkinson, H., Yoder, J. (2011). A Preliminary Exploration of the Effects of a 6-week Interactive Video Dance Exercise Program in an Adult Population. Cardiopulmonary Physical Therapy Journal, 22 (4), 5-11.

Miller, C.A., Hayes, D.M., Dye, K., Johnson, C., Meyers, J. (2012). Using the Nintendo Wii Fit and body weight support to improve aerobic capacity, balance, gait ability, and fear of falling: two case reports. Journal of Geriatric Physical Therapy, 35 (2), 95-104.

Mynarski, W., Nawrocka, A., Rozpara, M., Cholewa, J., Tomik, R. (2013). Objective methods, techniques and tools in physical activities testing. J. Tour. Recr. Sport Manag, 1, 63-78.

Noah, J.A., Spierer, D.K., Tachibana, A., Bronner, S. (2011). Vigorous energy expenditure with a dance exer-game. Journal of Exercise Physiology Online, 14 (4), 13-28.

Pichierri, G., Murer, K., de Bruin, E.D. (2012). A cognitive-motor intervention using a dance video game to enhance foot placement accuracy and gait under dual task conditions in older adults: a randomized controlled trial. BMC Geriatrics, 12 (1), 1. 
Polechonski, J., Mynarski, W., Nawrocka, A. (2015). Applicability of pedometry and accelerometry in the calculation of energy expenditure during walking and Nordic walking among women in relation to their exercise heart rate. J Phys Ther Sci, 27 (11), 3525-3527. DOI: 10.1589/jpts.27.3525.

Salonini, E., Gambazza, S., Meneghelli, I., Tridello, G., Sanguanini, M., Cazzarolli, C., Zanini, A., Assael, B.M. (2015). Active Video Game Playing in Children and Adolescents With Cystic Fibrosis: Exercise or Just Fun? Respiratory Care, 60 (8), 1172-1179. DOI: 10.4187/respcare.03576.

Schutz, Y., Weinsier, R.L., Hunter, G.R. (2001). Assessment of free-living physical activity in humans: an overview of currently available and proposed new measures. Obesity Research, 9 (6), 368-379. DOI: 10.1038/oby.2001.48.

Smallwood, S.R., Morris, M.M., Fallows, S.J., Buckley, J.P. (2012). Physiologic responses and energy expenditure of kinect active video game play in schoolchildren. Archives of Pediatrics and Adolescent Medicine, 166 (11), 1005-1009. DOI: 10.1001/ archpediatrics.2012.1271.

Smith, S.T., Sherrington, C., Studenski, S., Schoene, D., Lord, S.R. (2011). A novel Dance Dance Revolution (DDR) system for in-home training of stepping ability: basic parameters of system use by older adults. British Journal of Sports Medicine, 45 (5), $441-445$. DOI: 10.1136/bjsm.2009.066845.

Strath, S.J., Kaminsky, L.A., Ainsworth, B.E., Ekelund, U., Freedson, P.S., Gary, R.A., Richardson, C.R., Smith, D.T., Swartz, A.M. (2013). Guide to the assessment of physical activity: Clinical and research applications: a scientific statement from the American Heart Association. Circulation, 128 (20), 2259-2279. DOI: 10.1161/01.cir.0000435708.67487.da.

Studenski, S., Perera, S., Hile, E., Keller, V., Spadola-Bogard, J., Garcia, J. (2010). Interactive video dance games for healthy older adults. Journal of Nutrition, Health and Aging, 14 (10), 850-852.

Taylor, L.M., Kerse, N., Frakking, T., Maddison, R. (2016). Active Video Games for Improving Physical Performance Measures in Older People: A Meta-analysis. Journal of Geriatric Physical Therapy. DOI: 10.1519/JPT.0000000000000078.

Tripette, J., Ando, T., Murakami, H., Yamamoto, K., Ohkawara, K., Tanaka, S., Miyachi, M. (2014). Evaluation of active video games intensity: comparison between accelerometer-based predictions and indirect calorimetric measurements. Technology and Health Care, 22 (2), 199-208. DOI: 10.3233/THC-140817.

Trout, J., Christie, B. (2007). Interactive video games in physical education. Journal of Physical Education, Recreation \& Dance, 78 (5), 29-45.

Tudor-Locke, C., Craig, C.L., Aoyagi, Y., Bell, R.C., Croteau, K.A., De Bourdeaudhuij, I., Ewald, B., Gardner, A.W., Hatano, Y., Lutes, L.D., Matsudo, S.M., Ramirez-Marrero, F.A., Rogers, L.Q., Rowe, D.A., Schmidt, M.D., Tully, M.A., Blair, S.N. (2011). How many steps/day are enough? For older adults and special populations. International Journal of Behavioral Nutrition and Physical Activity, 8 (1), 1.

WHO (2010). Global recommendations on physical activity for health.

Cite this anticle aS:. Polechoński, J., Mynarski, W., Garbaciak, W., Fredyk, A., Rozpara, M., Nawrocka, A. (2018). Energy Expenditure and Intensity of Interactive Video Dance Games according to Health Recommendations. Central European Journal of Sport Sciences and Medicine, 4 (24), 35-43. DOI: 10.18276/cej.2018.4-04. 